Tohoku Math. J.

55 (2003), 175-188

\title{
DISTRIBUTIONS ON RIEMANNIAN MANIFOLDS, WHICH ARE HARMONIC MAPS
}

\author{
BOO-YONG CHOI AND JIN-WHAN YIM
}

(Received April 23, 2001)

\begin{abstract}
We find new examples of harmaonic maps between compact Riemannian manifolds. A section of a Riemannian fibration is called harmonic if it is harmonic as a map from the base manifold into the total space. When the fibres are totally geodesic, the EulerLagrange equation for such sections is formulated. In the case of distributions, which are sections of a Grassmannian bundle, this formula is described in terms of the geometry of base manifolds. Examples of harmonic distributions are constructed when the base manifolds are homogeneous spaces and the integral submanifolds are totally geodesic. In particular, we show all the generalized Hopf-fibrations define harmonic maps into the Grassmannian bundles with the standard metric.
\end{abstract}

1. Introduction. Let $M$ and $N$ be complete Riemannian manifolds. Assume $M$ is compact. A smooth map $f: M \rightarrow N$ is called harmonic if it is a critical point of the energy functional. If $N$ has non-positive curvature, then there exists a harmonic map in each homotopy class. However, if $N$ has positive curvature, this is no longer true and it always has been meaningful to find examples of harmonic map between compact manifolds with nonnegative curvature. In this paper, we produce some new type of harmonic maps between compact Riemannian manifolds.

We consider the case when $N$ is a fibre bundle over $M$ and $f: M \rightarrow N$ is a smooth map which happens to be a section of this fibration. If $N$ is a vector bundle over $M$ with a connection type metric, then $f$ is a harmonic map if and only if it is a parallel section. We will consider the case when the fibres are totally geodesic compact submanifolds, and hence $N$ is also a compact Riemannian manifold. In particular, if $N$ is a Grassmannian bundle associated to the orthogonal bundle $O(M)$, then the map $f$ defines a smooth distribution on $M$. A distribution on a Riemannian manifold $M$ will be called harmonic if it is harmonic as a map from $M$ into the total space of the Grassmannian bundle over $M$. We will find the Euler-Lagrange equation of a harmonic map $f$ in a general setting and find a solution to this equation to construct examples of harmonic distributions.

The definition of harmonic distributions, or harmonic sections in general, is used by other authors [7], [8], [9], [13] in a similar context but for a different concept. They call a section harmonic if its vertical energy is stationary with respect to vertical variations. If one is looking for a better section, this notion of harmonic section makes more sense. A variation

2000 Mathematics Subject Classification. Primary 53C30; Secondary 53C43, 58E20.

Key words and phrases. Harmonic map, distribution, homogeneous space.

Partially supported by KOSEF 99-2-102-002-3 and BK21 project, KAIST. 
through sections is necessarily a vertical one and it is easy to see that the horizontal energy does not change by the vertical variations. Therefore this vertically harmonic section will be a critical point of energy functional among smooth sections. In our case, however, we are more interested in the harmonic map itself, and therefore we have to consider both vertical and horizontal parts.

In [6], it is proved that a unit vector field on the round sphere $S^{3}$ is a harmonic map into its unit tangent bundle if and only if it is tangent to the Hopf-fibration. In this case, a unit vector field is considered as a one-dimensional distribution. This result was improved by Gluck and $\mathrm{Gu}$ [5] that a unit vector field is tangent to the Hopf-fibration if it is stationary only for the horizontal variations. They also gave an interesting interpretation of such horizontally harmonic vector fields on the standard 3-sphere that it corresponds to the so-called Beltrami field in fluid mechanics. This horizontal harmonicity apparently carries more geometry of manifold, but its geometric meaning is not yet well-understood.

In the following sections, we will consider sections of a fibre bundle with totally geodesic fibres, and find a formula of tension fields for sections as maps into the total space. In the case of Grassmannian bundles, this tension field will be written in terms of geometry of the base manifold. In the last section we will provide examples of harmonic distributions on Riemannian manifolds. The only known example on which the horizontal distribution is completely understood is the round sphere $S^{3}$ we mentioned above. When a vector field is harmonic on $S^{3}$, it is invariant under $S U(2)$-action and its integral curves are necessarily geodesics. Since it is extremely difficult to find a harmonic map for general Riemannian manifolds, we follow this line of reasoning, and study invariant distributions on homogeneous spaces with totally geodesic integral submanifolds. There is a standard way of constructing such manifolds. In fact, for compact Lie groups $K \subset H \subset G$ with suitable metric, the natural fibration

$$
\pi: M=G / K \rightarrow G / H
$$

has the totally geodesic fibre $H / K$. It is known that some homogeneous Einstein spaces can be constructed in this way, and in particular this construction includes distributions tangent to fibres in all of the generalized Hopf-fibrations. We will be able to show that all the generalized Hopf-fibrations define harmonic distributions in our sense.

We refer to [2], [3], [12] for basic tools and more detailed description of harmonic maps between Riemannian manifolds.

2. Harmonicity of sections. In this section, we will describe the Euler-Lagrange equation for the harmonic map, which is a section of a Riemannian fibration with totally geodesic fibres.

Let $M$ and $N$ be complete Riemannian manifolds. Assume $M$ is compact. A smooth map $\pi: N \rightarrow M$ is called a Riemannian submersion if $\pi$ is a submersion and for each $x \in N$, the horizontal subspace of $T_{x} N$ (orthogonal to the fibre over $\pi(x)$ in $N$ ) is mapped isometrically by $\left.d \pi\right|_{x}$ to $T_{\pi(x)} M$. We denote by $\mathcal{H}$ and $\mathcal{V}$ the horizontal and the vertical 
distribution, respectively. Then we can decompose the tangent bundle $T N=T N^{\mathcal{H}} \oplus T N^{\mathcal{V}}$, where we denote by $T N^{\mathcal{H}}$ and $T N^{\mathcal{V}}$ the horizontal and the vertical subbundle, respectively.

We now consider a Riemannian submersion with totally geodesic fibre $F$, that is, for each $x$ in $N$ with $p=\pi(x), \pi^{-1}(p)=F_{x}$ is a totally geodesic submanifold of $N$. Then all the fibres are isometric to each other and $\pi$ is a Riemannian fibration [1]. Furthermore, the horizontal distribution defines a connection on this fibre bundle. Let $f: M \rightarrow N$ be a smooth map which happens to be a section. The section $f$ is a harmonic map if and only if it is a critical point of the energy functional $E(f)=\int_{M} e(f) d v$, where $e(f)=(1 / 2)\|d f\|^{2}$ is the energy density of $f$. The differential map $d f$ is a differential 1-form with values in the pull-back bundle $f^{-1}(T N)$ and hence a section of $T^{*} M \otimes f^{-1}(T N)$. Decompose $f^{-1}(T N)$ as $f^{-1}\left(T N^{\mathcal{H}}\right) \oplus f^{-1}\left(T N^{\mathcal{V}}\right)$. We then have $d f=d f^{\mathcal{H}}+d f^{\mathcal{V}}$, where $d f^{\mathcal{H}} \in \Gamma\left(T^{*} M \otimes\right.$ $\left.f^{-1}\left(T N^{\mathcal{H}}\right)\right), d f^{\mathcal{V}} \in \Gamma\left(T^{*} M \otimes f^{-1}\left(T N^{\mathcal{V}}\right)\right)$, and $\Gamma(\cdot)$ denotes the set of all smooth sections of the corresponding bundle. Then the energy $E(f)$ is given by

$$
E(f)=E^{\mathcal{H}}(f)+E^{\mathcal{V}}(f)=\frac{1}{2} \int_{M}\left\|d f^{\mathcal{H}}\right\|^{2} d v+\frac{1}{2} \int_{M}\left\|d f^{\mathcal{V}}\right\|^{2} d v
$$

Since $f$ is a section of a Riemannian fibration, the linear map $d f_{p}^{\mathcal{H}}: T_{p} M \rightarrow\left(T_{x} N\right)^{\mathcal{H}}$ is an isometry for each $p=\pi(x)$, and hence we have $E^{\mathcal{H}}(f)=(m / 2) \operatorname{Vol}(M)(m=\operatorname{dim}(M))$.

In the case of a vector bundle with metric connection, it is easy to see that a section is harmonic if and only if it is parallel. In fact, for a section $f: M \rightarrow N$, consider a variation of $f$ given by $f_{t}(p)=t f(p), p \in M$. For an orthonormal basis $\left\{e_{i}\right\}$ of $T_{p} M$, $d f\left(e_{i}\right)=\tilde{e}_{i}+\nabla_{e_{i}} f$, where $\tilde{e}_{i}$ is the horizontal lift of $e_{i}$. Hence $d f_{t}\left(e_{i}\right)=\tilde{e}_{i}+t \nabla_{e_{i}} f$, and

$$
E\left(f_{t}\right)=\frac{1}{2} \int_{M}\left\|d f_{t}\right\|^{2} d v=\frac{1}{2}\left(m \operatorname{Vol}(M)+t^{2} \int_{M}\left\|\nabla_{e_{i}} f\right\|^{2} d v\right) .
$$

Therefore $\left.(d / d t)\right|_{t=1} E\left(f_{t}\right)=0$ implies $\nabla f \equiv 0$ (In the case of tangent bundle, see [9].).

For $f: M \rightarrow N$ we now consider the Euler-Lagrange equation of the energy functional. Let $\nabla$ and $\tilde{\nabla}$ be the Levi-Civita connection on $M$ and $N$, respectively, and let $\bar{\nabla}$ be the induced connection on the pull-back bundle $f^{-1}(T N)$. Then we have $\bar{\nabla}(d f) \in \Gamma\left(\left(S^{2} M \otimes\right.\right.$ $\left.\left.f^{-1}\left(T N^{\mathcal{H}}\right)\right) \oplus\left(S^{2} M \otimes f^{-1}\left(T N^{\mathcal{V}}\right)\right)\right)$, where $S^{2} M$ is the space of symmetric covariant 2tensors. Taking trace of the second fundamental form gives the tension field,

$$
\tau(f)=-\bar{\nabla}^{*}(d f)=\operatorname{Tr}(\bar{\nabla} d f) \in \Gamma\left(f^{-1}(T N)\right) .
$$

Then $f: M \rightarrow N$ is a harmonic map if and only if $\tau(f)=0$. In fact, for a vector field $V$ along $f$, let $\Sigma:(-\varepsilon, \varepsilon) \times M \rightarrow N$ be a variation such that $\Sigma(0, p)=f(p),(\partial \Sigma / \partial t)(0, p)=$ $V(p)$, and $f_{t}(p):=\Sigma(t, p)$. Then

$$
\left.\frac{d}{d t}\right|_{t=0} E\left(f_{t}\right)=-\int_{M}\langle V, \tau(f)\rangle_{N} d v
$$

where $\langle\cdot, \cdot\rangle_{N}$ denotes the Riemannian metric on $N$. We decompose $\tau(f)$ as $\tau(f)=\tau^{\mathcal{H}}(f)+$ $\tau^{\mathcal{V}}(f)$, where $\tau^{\mathcal{H}}(f) \in \Gamma\left(f^{-1}\left(T N^{\mathcal{H}}\right)\right)$ and $\tau^{\mathcal{V}}(f) \in \Gamma\left(f^{-1}\left(T N^{\mathcal{V}}\right)\right)$. Then we have 


$$
\begin{aligned}
\tau^{\mathcal{H}}(f) & =\left(\operatorname{Tr} \bar{\nabla} d f^{\mathcal{H}}\right)^{\mathcal{H}}+\left(\operatorname{Tr} \bar{\nabla} d f^{\mathcal{V}}\right)^{\mathcal{H}}, \\
\tau^{\mathcal{V}}(f) & =\left(\operatorname{Tr} \bar{\nabla} d f^{\mathcal{H}}\right)^{\mathcal{V}}+\left(\operatorname{Tr} \bar{\nabla} d f^{\mathcal{V}}\right)^{\mathcal{V}} .
\end{aligned}
$$

LEMMA 1. For a smooth section $f: M \rightarrow N$, we have

$$
\left(\operatorname{Tr} \bar{\nabla} d f^{\mathcal{H}}\right)^{\mathcal{V}}=0, \quad\left(\operatorname{Tr} \bar{\nabla} d f^{\mathcal{V}}\right)^{\mathcal{H}}=\left(\operatorname{Tr} \bar{\nabla} d f^{\mathcal{H}}\right)^{\mathcal{H}} .
$$

Proof. For a vector field $X$ on $M$ let $\tilde{X}$ denote the basic vector field which is a horizontal lift of $X$. Then for a local orthonormal frame field $\left\{e_{i}\right\}$ of $M, d f^{\mathcal{H}}\left(e_{i}\right)=\tilde{e}_{i}$ and

$$
\operatorname{Tr} \bar{\nabla} d f^{\mathcal{H}}=\sum_{i}\left(\bar{\nabla}_{e_{i}} d f^{\mathcal{H}}\right)\left(e_{i}\right)=\sum_{i}\left(\bar{\nabla}_{e_{i}} \tilde{e}_{i}-\widetilde{\left(\nabla_{e_{i}} e_{i}\right)}\right) \text {. }
$$

Since $\bar{\nabla}_{e_{i}} \tilde{e}_{i}=\tilde{\nabla}_{\tilde{e}_{i}} \tilde{e}_{i}+\tilde{\nabla}_{d f} \mathcal{V}_{\left(e_{i}\right)} \tilde{e}_{i}$ and $\left.\tilde{\nabla}_{\tilde{e}_{i}} \tilde{e}_{i}=\widetilde{\left(\nabla_{e_{i}} e_{i}\right.}\right)$ by O'Neill's formula ([10]), we have $\operatorname{Tr} \bar{\nabla} d f^{\mathcal{H}}=\sum_{i} \tilde{\nabla}_{d f} \mathcal{V}_{\left(e_{i}\right)} \tilde{e}_{i}$. Since fibres are totally geodesic, we have $\tilde{\nabla}_{d f} \mathcal{V}_{\left(e_{i}\right)} \tilde{e}_{i} \in \mathcal{H}$, and hence

$$
\left(\operatorname{Tr} \bar{\nabla} d f^{\mathcal{H}}\right)^{\mathcal{V}}=0, \quad\left(\operatorname{Tr} \bar{\nabla} d f^{\mathcal{H}}\right)^{\mathcal{H}}=\sum_{i} \tilde{\nabla}_{d f^{\mathcal{V}}\left(e_{i}\right)} \tilde{e}_{i} .
$$

For the vertical component, we locally extend $d f^{\mathcal{V}}\left(e_{i}\right)$, a vector field along $f$, to a vertical vector field on $N$, which we also denote by $d f^{\mathcal{V}}\left(e_{i}\right)$. We then have

$$
\begin{aligned}
\operatorname{Tr} \bar{\nabla} d f^{\mathcal{V}} & =\sum_{i}\left(\bar{\nabla}_{e_{i}} d f^{\mathcal{V}}\right)\left(e_{i}\right)=\sum_{i}\left(\bar{\nabla}_{e_{i}} d f^{\mathcal{V}}\left(e_{i}\right)-d f^{\mathcal{V}}\left(\nabla_{e_{i}} e_{i}\right)\right) \\
& =\sum_{i}\left(\tilde{\nabla}_{\tilde{e}_{i}} d f^{\mathcal{V}}\left(e_{i}\right)+\tilde{\nabla}_{d f} \mathcal{V}\left(e_{i}\right)\right. \\
& \left.d f^{\mathcal{V}}\left(e_{i}\right)-d f^{\mathcal{V}}\left(\nabla_{e_{i}} e_{i}\right)\right),
\end{aligned}
$$

where $d f^{\mathcal{V}}\left(\nabla_{e_{i}} e_{i}\right)$ and $\tilde{\nabla}_{d f} \mathcal{V}_{\left(e_{i}\right)} d f^{\mathcal{V}}\left(e_{i}\right)$ are in $\mathcal{V}$ because the fibres are totally geodesic. Furthermore, since $\tilde{e}_{i}$ is a basic vector field and $d f^{\mathcal{V}}\left(e_{i}\right)$ is vertical, we have $\left[\tilde{e}_{i}, d f^{\mathcal{V}}\left(e_{i}\right)\right] \in \mathcal{V}$. Therefore

$$
\left(\bar{\nabla}_{e_{i}} d f^{\mathcal{V}}\left(e_{i}\right)\right)^{\mathcal{H}}=\left(\tilde{\nabla}_{\tilde{e}_{i}} d f^{\mathcal{V}}\left(e_{i}\right)\right)^{\mathcal{H}}=\tilde{\nabla}_{d f} \mathcal{V}_{\left(e_{i}\right)} \tilde{e}_{i} .
$$

Thus we conclude that $\left(\operatorname{Tr} \bar{\nabla} d f^{\mathcal{V}}\right)^{\mathcal{H}}=\sum_{i} \tilde{\nabla}_{d f} \mathcal{V}_{\left(e_{i}\right)} \tilde{e}_{i}=\left(\operatorname{Tr} \bar{\nabla} d f^{\mathcal{H}}\right)^{\mathcal{H}}$.

Denote by $\mathcal{H}_{\bar{\nabla}}$ and $\mathcal{V} \bar{\nabla}$ the horizontal and the vertical component of $\bar{\nabla}$, respectively. Since $\operatorname{Tr}\left({ }^{\mathcal{H}} \bar{\nabla} d f^{\mathcal{H}}\right)=\left(\operatorname{Tr} \bar{\nabla} d f^{\mathcal{H}}\right)^{\mathcal{H}}$ and $\operatorname{Tr}\left({ }^{\mathcal{V}} \bar{\nabla} d f^{\mathcal{V}}\right)=\left(\operatorname{Tr} \bar{\nabla} d f^{\mathcal{V}}\right)^{\mathcal{V}}$, we use the rough Laplacian notation of the induced connections.

Definition 1. For $f: M \rightarrow N$ as above, we denote

$$
\begin{aligned}
& \Delta^{\mathcal{H}}(f)={ }^{\mathcal{H}} \bar{\nabla}^{*}\left(d f^{\mathcal{H}}\right)=-\operatorname{Tr}\left({ }^{\mathcal{H}} \bar{\nabla} d f^{\mathcal{H}}\right), \\
& \Delta^{\mathcal{V}}(f)={ }^{\mathcal{V}} \bar{\nabla}^{*}\left(d f^{\mathcal{V}}\right)=-\operatorname{Tr}\left({ }^{\mathcal{V}} \bar{\nabla} d f^{\mathcal{V}}\right) .
\end{aligned}
$$

Then Lemma 1 implies the following theorem. 
THEOREM 1. For $f: M \rightarrow N$ as above, we have

$$
\tau(f)=\tau^{\mathcal{H}}(f)+\tau^{\mathcal{V}}(f)=-\left(2 \Delta^{\mathcal{H}}(f)+\Delta^{\mathcal{V}}(f)\right) .
$$

This result is intriguing in the sense that the vertical tension coincides with the vertical Laplacian, but the horizontal one has the factor 2. Nonetheless, $f$ is harmonic if and only if it is harmonic with respect to both Laplacians.

Since $E(f)=E^{\mathcal{H}}(f)+E^{\mathcal{V}}(f)$ and $E^{\mathcal{H}}(f)$ is constant as long as $f$ is a section, we see that $\tau^{\mathcal{V}}(f)=0$ if and only if $E^{\mathcal{V}}(f)$ is stationary with respect to vertical variations (compare [13], Theorem 2). The horizontal tension field along $f$ is a horizontal lift of a vector field on $M$, and it apparently carries more geometry of $M$ than the vertical one. In the remainder of this section we will try to find a more geometric description of the horizontal tension fields.

Let $\bar{\pi}: P \rightarrow M$ be the principal $G$-bundle associated to $N, F$ the fibre space on which $G$ acts, and $\varphi: P \times F \rightarrow N$ the principal map. Let $\omega$ and $\Omega$ be the corresponding connection form and the curvature form on $P$. For $p \in M$, choose $(u, \xi) \in P \times F$ such that $\varphi(u, \xi)=$ $f(p)$. We then fix $\xi$ and consider the map $\varphi_{\xi}: P \rightarrow N$ defined by $\varphi_{\xi}(\alpha)=\varphi(\alpha, \xi)$. The connection on $N$ is associated to that of $P$, and therefore the horizontal space of $N$ is, by definition, the image of the horizontal space of $P$ by $\varphi_{\xi}$. Let $\bar{e}_{i}$ be the horizontal vector field on $P$ such that $d \varphi_{\xi}\left(\bar{e}_{i}\right)=\tilde{e}_{i}$. Then by the structure equation we have $\omega_{u}\left(\left[\bar{e}_{i}, \bar{e}_{j}\right]\right)=$ $-\Omega_{u}\left(\bar{e}_{i}, \bar{e}_{j}\right)$, and hence

$$
\left[\tilde{e}_{i}, \tilde{e}_{j}\right]^{\mathcal{V}}=d \varphi_{\xi}\left(\left[\bar{e}_{i}, \bar{e}_{j}\right]^{\mathcal{V}}\right)=-d \varphi_{\xi}\left(\left(\omega_{u} \mid \mathcal{V}\right)^{-1}\left(\Omega_{u}\left(\bar{e}_{i}, \bar{e}_{j}\right)\right)\right)
$$

We use this fact to prove the following proposition.

Proposition 1. Let $\pi: N \rightarrow M$ be a Riemannian submersion with totally geodesic fibres and $f: M \rightarrow N$ a section. Then

$$
\tau^{\mathcal{H}}(f)=\sum_{i, j}\left\langle d \varphi_{\xi}\left(\left(\omega_{u} \mid \mathcal{V}\right)^{-1}\left(\Omega_{u}\left(\bar{e}_{i}, \bar{e}_{j}\right)\right)\right), d f^{\mathcal{V}}\left(e_{i}\right)\right\rangle_{N} \tilde{e}_{j},
$$

where $\left\{\tilde{e}_{i}\right\}$ and $\left\{\bar{e}_{i}\right\}$ are the horizontal lifts to $P$ and $N$ of an orthonormal frame field $\left\{e_{i}\right\}$ of $M$.

PROOF. For a local orthonormal frame field $\left\{e_{i}\right\}$ on $M$, by Lemma 1, we have

$$
\begin{aligned}
\Delta^{\mathcal{H}}(f) & =-\sum_{i}\left(\tilde{\nabla}_{\tilde{e}_{i}} d f^{\mathcal{V}}\left(e_{i}\right)\right)^{\mathcal{H}}=-\sum_{i, j}\left\langle\tilde{\nabla}_{\tilde{e}_{i}} d f^{\mathcal{V}}\left(e_{i}\right), \tilde{e}_{j}\right\rangle_{N} \tilde{e}_{j} \\
& =\sum_{i, j}\left\langle d f^{\mathcal{V}}\left(e_{i}\right), \tilde{\nabla}_{\tilde{e}_{i}} \tilde{e}_{j}\right\rangle_{N} \tilde{e}_{j} .
\end{aligned}
$$

Since $\tilde{\nabla}_{\tilde{e}_{i}} \tilde{e}_{j}=\left(\tilde{\nabla}_{\tilde{e}_{i}} \tilde{e}_{j}\right)^{\mathcal{H}}+A_{\tilde{e}_{i}} \tilde{e}_{j}$, where $A$ is the O'Neill tensor (see [10]) and $A_{\tilde{e}_{i}} \tilde{e}_{j}=$ $(1 / 2)\left[\tilde{e}_{i}, \tilde{e}_{j}\right]^{\mathcal{V}}$ for horizontal vector fields, we have

$$
\Delta^{\mathcal{H}}(f)=\frac{1}{2} \sum_{i, j}\left\langle\left[\tilde{e}_{i}, \tilde{e}_{j}\right]^{\mathcal{V}}, d f^{\mathcal{V}}\left(e_{i}\right)\right\rangle_{N} \tilde{e}_{j} .
$$

Then the proposition follows from Theorem 1 and the above remark. 
3. Distributions on Riemannian manifolds. In this section, we apply the results in Section 2 to smooth distributions on Riemannian manifolds. We note that in general it is not very difficult to find an equation for harmonic maps between two Riemannian manifolds as long as Riemannian metrics are given. In fact, an explicit formula for the tension field of distributions in terms of local coordinates appears in [14] (This result has not been published to the best of author's knowledge), and the vertical component was also studied in [13]. For the sake of completeness, we will show how they can be derived from the general formula we found in the previous section. We also need the equation in a specific form so that we can find solutions in the next section.

A $k$-dimensional distribution on an $n$-dimensional manifold $M$ is a smooth section of the Grassmannian bundle $G_{k}(M)$ of $k$-dimensional planes in tangent spaces of $M$. Throughout this section we use the index convention,

$$
1 \leq i, j \leq k, \quad k+1 \leq \alpha, \beta \leq n, \quad 1 \leq A, B \leq n
$$

The Grassmannian bundle $G_{k}(M)$ is associated to the orthogonal bundle $O(M)$, which is a principal $O(n)$-bundle, and has the fibre $G_{k}\left(\boldsymbol{R}^{n}\right)=O(n) /(O(k) \times O(n-k))(=G / K)$. An invariant metric on $G_{k}\left(\boldsymbol{R}^{n}\right)$ is determined by an $\operatorname{Ad}_{G}(K)$-invariant inner product on a subspace $\mathfrak{m} \subset \mathfrak{o}(n)$, which is an $\operatorname{Ad}_{G}(K)$-invariant complement to $(\mathfrak{o}(k) \times \mathfrak{o}(n-k))$ and is identified with $T_{(e K)} G_{k}\left(\boldsymbol{R}^{n}\right)$ by the projection map. Let $E_{B}^{A}$ denote the $(n \times n)$-matrix such that $(A, B)$-th entry is 1 and others are all zero. Then for the standard metric on $G_{k}\left(\boldsymbol{R}^{n}\right)$, we take $\mathfrak{m}\left(\boldsymbol{R}^{n}\right)$ as a subspace with the orthonormal basis $\left\{e_{\alpha}^{i}:=E_{i}^{\alpha}-E_{\alpha}^{i}\right\}$.

Consider the principal map $\varphi: O(M) \times G_{k}\left(\boldsymbol{R}^{n}\right) \rightarrow G_{k}(M)$. Let $\xi$ be the origin of $G_{k}\left(\boldsymbol{R}^{n}\right)$, i.e., the coset $O(k) \times O(n-k)$. Then $\varphi_{\xi}: O(M) \rightarrow G_{k}(M)$ is nothing but the projection $O\left(T_{p} M\right) \rightarrow G_{k}\left(T_{p} M\right)$ on fibre over each $p \in M$, and $d \varphi_{\xi}: \mathfrak{o}\left(T_{p} M\right) \rightarrow \mathfrak{m}\left(T_{p} M\right)$ is the corresponding projection in Lie algebra.

Let $\mathcal{D}$ be a $k$-dimensional distribution on $M$, which we also denote by a map $f: M \rightarrow$ $G_{k}(M)$. Denote by $\mathcal{D}^{\perp}$ the orthogonal complement to $\mathcal{D}$. Choose a local orthonormal frame field $\left\{e_{A}\right\}$ on an open subset $U$ of $M$ such that

$$
e_{i} \in \mathcal{D}, \quad 1 \leq i \leq k, \quad e_{\alpha} \in \mathcal{D}^{\perp}, \quad k+1 \leq \alpha \leq n
$$

Then this frame field defines a local section $\sigma: U \rightarrow O(U)$ such that $\varphi_{\xi} \circ \sigma=\left.f\right|_{U}$. With respect to this frame field, we can locally trivialize $O(U)$ as $U \times O(n)$, and through $\varphi_{\xi}$ we obtain a local trivialization $U \times G_{k}\left(\boldsymbol{R}^{n}\right)$ of $G_{k}(U)$. Then the distribution $\mathcal{D}$ appears as the origin in $G_{k}\left(\boldsymbol{R}^{n}\right)$. Let $\omega$ and $\Omega$ be the connection form and curvature form of this principal bundle. For $p \in M$ let $u \in O(M)$ denote the orthonormal basis $\left\{e_{A}\right\}$ of $T_{p} M$. Then for $v \in T_{p} M, d \sigma^{\mathcal{V}}(v)=\left(\omega_{u} \mid \mathcal{V}\right)^{-1}\left(\omega_{u}(d \sigma(v))\right.$ is a skew-symmetric matrix whose $(A, B)$ th entry is given by $\left\langle\nabla_{v} e_{B}, e_{A}\right\rangle$, where $\langle\cdot, \cdot\rangle$ is the metric of $M$. Note that with respect to our trivialization, $\left(\omega_{u} \mid \mathcal{V}\right)$ is simply the identification of the tangent space of $O(n)$ to its Lie algebra. We now improve Proposition 1 to have a more explicit formula for the horizontal tension field of a distribution. We denote by $(\cdot)^{\top}$ and $(\cdot)^{\perp}$ the projections onto $\mathcal{D}$ and $\mathcal{D}^{\perp}$, respectively. 
PROPOSITION 2. For a distribution $\mathcal{D}$ on $M$, let $\left\{e_{A}\right\}$ be a local orthonormal frame field such that $e_{i} \in \mathcal{D}$. Then

$$
\tau^{\mathcal{H}}(\mathcal{D})=\sum_{i, A} R\left(\left(\nabla_{e_{A}} e_{i}\right)^{\perp}, e_{i}\right) e_{A}
$$

where $R$ is the curvature tensor of $M$, and $\tau^{\mathcal{H}}(\mathcal{D})$ is identified with its image on $M$ by the isometry $d f^{\mathcal{H}}: T_{p} M \rightarrow T_{f(p)} N^{\mathcal{H}}$.

PROOF. Since $d \varphi_{\xi}: \mathfrak{o}\left(T_{p} M\right) \rightarrow \mathfrak{m}\left(T_{p} M\right)$ is a projection such that $d f^{\mathcal{V}}(v)=$ $d \varphi_{\xi}\left(d \sigma^{\mathcal{V}}(v)\right)$, we have

$$
d f^{\mathcal{V}}(v)=\sum_{i, \alpha}\left\langle\nabla_{v} e_{i}, e_{\alpha}\right\rangle E_{i}^{\alpha}+\left\langle\nabla_{v} e_{\alpha}, e_{i}\right\rangle E_{\alpha}^{i}=\sum_{i, \alpha}\left\langle\nabla_{v} e_{i}, e_{\alpha}\right\rangle e_{\alpha}^{i} .
$$

Furthermore, for the horizontal lift $\left\{\bar{e}_{A}\right\}$ of $\left\{e_{A}\right\}$ in $O(M)$, we see that $\left(\omega_{u} \mid \mathcal{V}\right)^{-1} \Omega_{u}\left(\bar{e}_{A}, \bar{e}_{B}\right)$ is the skew-symmetric endomorphism $R\left(e_{A}, e_{B}\right): T_{p} M \rightarrow T_{p} M$, and hence

$$
d \varphi_{\xi}\left(\left(\omega_{u} \mid \mathcal{V}\right)^{-1} \Omega_{u}\left(\bar{e}_{A}, \bar{e}_{B}\right)\right)=\sum_{i, \alpha}\left\langle R\left(e_{A}, e_{B}\right) e_{i}, e_{\alpha}\right\rangle e_{\alpha}^{i} .
$$

Then by Proposition 1 and the fact that $\left\{e_{\alpha}^{i}\right\}$ is an orthonormal basis of $T_{p} M$, which is identified with $\mathfrak{m}$, we have

$$
\tau^{\mathcal{H}}(\mathcal{D})=\sum_{i, A, B}\left\langle\left(R\left(e_{A}, e_{B}\right) e_{i}\right)^{\perp},\left(\nabla_{e_{B}} e_{i}\right)^{\perp}\right\rangle e_{A} .
$$

Then the proposition follows from the symmetry of the curvature tensor.

As an example, we consider the case when $M=S^{n}$ is a round sphere with constant curvature $K>0$ and $\mathcal{D}$ is a unit vector field $\xi$. Then the curvature tensor $R$ is given by $R(X, Y) Z=K(\langle X, Z\rangle Y-\langle Y, Z\rangle X)$. Choose an orthonormal frame $\left\{e_{A}\right\}, i=1, \ldots, n$. Since $\left\langle\nabla_{v} \xi, \xi\right\rangle=0$ for any vector $v$, the horizontal tension field of $\xi$ is

$$
\begin{aligned}
\tau^{\mathcal{H}}(\xi) & =\sum_{A=1}^{n} R\left(\left(\nabla_{e_{A}} \xi\right)^{\perp}, \xi\right) e_{A} \\
& =K \sum_{A=1}^{n}\left(\left\langle\nabla_{e_{A}} \xi, e_{A}\right\rangle \xi-\left\langle\xi, e_{A}\right\rangle \nabla_{e_{A}} \xi\right) \\
& =K\left((\operatorname{div} \xi) \xi-\nabla_{\xi} \xi\right),
\end{aligned}
$$

where $\operatorname{div} \xi$ denotes the divergence of the vector field $\xi$. Therefore $\xi$ is horizontally harmonic if and only if $\operatorname{div} \xi=\nabla_{\xi} \xi=0$, which means the flow is volume preserving and the integral curves are geodesics.

The vertical tension field for distributions has been studied extensively [13] and we will only mention a few facts. From the equation for $d f^{\mathcal{V}}(v)$ in the above proof, it is now easy to see that for any $X \in T_{p} M$, we have (compare [13], Theorem 3)

$$
d f^{\mathcal{V}}(v)(X)=P_{v}(X)+Q_{v}(X):=\left(\nabla_{v} X^{\top}\right)^{\perp}+\left(\nabla_{v} X^{\perp}\right)^{\top},
$$


where $P_{v}(X)$ and $Q_{v}(X)$ are independent of any extension of $X$ to a vector field and hence $d f^{\mathcal{V}}(v)$ is a tensor. Moreover $\left.P_{v}\right|_{\mathcal{D}} \in \operatorname{Hom}\left(\mathcal{D}, \mathcal{D}^{\perp}\right)$ and $\left.Q_{v}\right|_{\mathcal{D}^{\perp}} \in \operatorname{Hom}\left(\mathcal{D}^{\perp}, \mathcal{D}\right)$ satisfy $\left.P_{v}\right|_{\mathcal{D}}=-\left(\left.Q_{v}\right|_{\mathcal{D}^{\perp}}\right)^{*}$.

In the case of Grassmannian bundles, the induced vertical connection ${ }^{\mathcal{V}} \bar{\nabla}$ applied to the vertical vector field $d f^{\mathcal{V}}\left(e_{A}\right)$ in fact coincides with the covariant derivative of the local tensor field $d f^{\mathcal{V}}\left(e_{A}\right)$. Since $\tau^{\mathcal{V}}(\mathcal{D}) \in \mathfrak{m}\left(T_{p} M\right)$, by the same argument as $d f^{\mathcal{V}}(v)$, we can decompose $\tau^{\mathcal{V}}(\mathcal{D})$. Therefore

$$
\tau^{\mathcal{V}}(\mathcal{D})=-{ }^{\mathcal{V}} \bar{\nabla}^{*}\left(d f^{\mathcal{V}}\right)=-\nabla^{*}\left(d f^{\mathcal{V}}\right):=P+Q,
$$

where $\left.P\right|_{\mathcal{D}} \in \operatorname{Hom}\left(\mathcal{D}, \mathcal{D}^{\perp}\right)$ and $\left.Q\right|_{\mathcal{D}^{\perp}}=-\left(\left.P\right|_{\mathcal{D}}\right)^{*} \in \operatorname{Hom}\left(\mathcal{D}^{\perp}, \mathcal{D}\right)$. It is then an easy computation of tensor derivation to see the following.

Proposition 3. Suppose that we identify $\mathfrak{m}\left(T_{p} M\right)$ as the space of skew-symmetric operators on $T_{p} M$, which can be decomposed as $\operatorname{Hom}\left(\mathcal{D}, \mathcal{D}^{\perp}\right) \oplus \operatorname{Hom}\left(\mathcal{D}^{\perp}, \mathcal{D}\right)$. Then for each $v \in \mathcal{D}$, we have

$$
\begin{aligned}
\tau^{\mathcal{V}}(\mathcal{D})(v) & =\left.P\right|_{\mathcal{D}}(v) \\
& =\sum_{A}\left(\left(\nabla_{e_{A}}\left(\nabla_{e_{A}} v\right)^{\perp}\right)^{\perp}-\left(\nabla_{e_{A}}\left(\nabla_{e_{A}} v\right)^{\top}\right)^{\perp}-\left(\nabla_{\nabla_{e_{A}} e_{A}} v\right)^{\perp}\right),
\end{aligned}
$$

and $\left.\tau^{\mathcal{V}}(\mathcal{D})\right|_{\mathcal{D}^{\perp}}=-\left(\left.\tau^{\mathcal{V}}(\mathcal{D})\right|_{\mathcal{D}}\right)^{*}$.

An immediate consequence of our discussion above is that we can obtain the tension field of the complementary distribution $\mathcal{D}^{\perp}$ by simply reordering the indices, and hence $\tau(\mathcal{D})=$ $\tau\left(\mathcal{D}^{\perp}\right)$.

4. Examples of harmonic distributions. In this section, we will find examples of Riemannian manifolds with harmonic distributions.

In [6], it is shown that on the 3-dimensional unit sphere, a smooth unit vector field is a harmonic map into the unit tangent bundle with the Sasaki metric if and only if it is the tangent vector field of the Hopf-fibration. A crucial fact about the Hopf vector field is that it is invariant under $S U(n+1)$ action on $S^{2 n+1}=S U(n+1) / S U(n)$ and the integral curves are geodesics. Here we note that in this case the metric on $S U(n+1)$ is not the standard biinvariant metric. In general, an irreducible symmetric space $G / K$ presented by a symmetric pair $(G, K)$ does not carry any $G$-invariant distribution.

Let $M=G / K$ be a reductive homogeneous space with transitive action by a Lie group $G$. Let $\mathfrak{g}$ be the Lie algebra of $G$ and $\mathfrak{k} \subset \mathfrak{g}$ the corresponding subalgebra for $K$, and choose an $\operatorname{Ad}_{G}(K)$-invariant complement $\mathfrak{m}$ to $\mathfrak{k}$ in $\mathfrak{g}$. A distribution $\mathcal{D}$ is called $G$-invariant if $d \gamma\left(\mathcal{D}_{p}\right)=\mathcal{D}_{\gamma(p)}$ for each $p \in M$ and $\gamma \in G$. It is then easy to see that by the isomorphism $\mathfrak{m} \rightarrow T_{(e K)} M$ there is a one-to-one correspondence between $\operatorname{Ad}_{G}(K)$-invariant subspaces of $\mathfrak{m}$ and $G$-invariant distributions on $M$.

In order to produce examples with harmonic distributions, we now recall a standard technique to construct homogeneous Riemannian fibration with totally geodesic fibres (see [1]). 
Let $G$ be a compact Lie group, $H, K$ two compact subgroups of $G$ with $K \subset H$. Then we have the natural fibration

$$
\pi: M=G / K \rightarrow G / H
$$

with fibre $H / K$. We further assume that $G / H$ is an irreducible symmetric space with the symmetric pair $(G, H)$. Let $\mathfrak{k} \subset \mathfrak{h} \subset \mathfrak{g}$ be the Lie algebras of $K, H$, and $G$, respectively. We choose an $\operatorname{Ad}_{G}(H)$-invariant complement $\mathfrak{n}$ to $\mathfrak{h}$ in $\mathfrak{g}$, and an $\operatorname{Ad}_{G}(K)$-invariant complement $\mathfrak{p}$ to $\mathfrak{k}$ in $\mathfrak{h}$. Then $\mathfrak{m}:=\mathfrak{p} \oplus \mathfrak{n}$ is an $\operatorname{Ad}_{G}(K)$-invariant complement to $\mathfrak{k}$ in $\mathfrak{g}$, and we have $[\mathfrak{h}, \mathfrak{n}] \subset \mathfrak{n},[\mathfrak{k}, \mathfrak{p}] \subset \mathfrak{p}$. Furthermore, since $(G, H)$ is a symmetric pair we have $[\mathfrak{n}, \mathfrak{n}] \subset \mathfrak{h}$. An $\operatorname{Ad}_{G}(H)$-invariant scalar product on $\mathfrak{n}$ defines a $G$-invariant Riemannian metric $\check{g}$ on $G / H$, and an $\operatorname{Ad}_{G}(K)$-invariant scalar product on $\mathfrak{p}$ defines a $H$-invariant Riemannian metric $\hat{g}$ on $H / K$. Finally, the orthogonal direct sum for these scalar products on $\mathfrak{m}=\mathfrak{p} \oplus \mathfrak{n}$ defines a $G$-invariant Riemannian metric $g$ on $G / K$. Then we see that the map $\pi$ is a Riemannian submersion from $(G / K, g)$ to $(G / H, \breve{g})$ with totally geodesic fibres isometric to $(H / K, \hat{g})$.

For example, let $G=S U(n+1), H=S(U(1) \times U(n))$ and $K=S U(n)$. Then we see that the corresponding fibration is $\pi: S^{2 n+1} \rightarrow C P^{n}$, which is called the Hopf-fibration. Notice that the standard $S U(n+1)$-invariant metric $g$ on $S^{2 n+1}$ is not the round metric. However by re-scaling the metric $g$ along the fibre we can produce the standard round sphere, and obtain an $S U(n+1)$-invariant distribution $\mathcal{D}$ tangent to the Hopf-fibration. In general, for the homogeneous spaces $M=G / K$ as above, we need to change the bi-invariant metric on $G$ in order to obtain interesting metrics on $M$. We show that this type of modification can be done in a general setting.

Let $\langle\langle\cdot, \cdot\rangle\rangle$ be a bi-invariant metric on $G$ and let $\langle\cdot, \cdot\rangle$ a new left invariant metric defined by re-scaling $\langle\langle\cdot, \cdot\rangle\rangle$ on $\mathfrak{h}$ such that $\left.\langle\cdot, \cdot\rangle\right|_{\mathfrak{h}}=\lambda\langle\langle\cdot, \cdot\rangle\rangle$ for a constant $\lambda>0$. It is easy to see that this new metric on $G$ is still $H$-biinvariant, and hence its restriction on $\mathfrak{m}$ is $\operatorname{Ad}_{G}(K)$-invariant. Therefore, it determines an invariant metric on $M=G / K$ that we also denote by $\langle\cdot, \cdot\rangle$. The projection $\pi: G \rightarrow M$ is a Riemannian submersion. By choosing $\lambda>0$, we produce a family of new metrics on $M$, and it is known that some homogeneous Einstein manifolds can be produced in this way [1]. In particular, this construction includes all of the generalized Hopf-fibrations. Since this new metric on $G$ is not bi-invariant and $M$ is not necessarily a normal homogeneous space, it takes some effort to understand the geometry of $M$ in terms of the algebraic structures.

Let $M=G / K$ be a homogeneous space with the metric $\langle\cdot, \cdot\rangle$ defined as above. The compact Lie group $G$ acts on $M$ by isometries and hence also acts on the Grassmannian bundle $G_{k}(M), k=\operatorname{dim}(\mathfrak{p})$, with the standard metric as in Section 3. The $\operatorname{Ad}_{G}(K)$-invariant space $\mathfrak{p}$ generates a $G$-invariant distribution on $M$, which we denote by $\mathcal{D}$, and hence $\mathcal{D}^{\perp}$ is generated by $\mathfrak{n}$. Since $\mathcal{D}$ is $G$-invariant, so is the tension vector field $\tau(\mathcal{D})$ on $G_{k}(M)$. Since the $G$-action on $G_{k}(M)$ clearly preserves the horizontal and vertical spaces, we see that $\tau^{\mathcal{V}}(\mathcal{D})$ and $\tau^{\mathcal{H}}(\mathcal{D})$ are both $G$-invariant. Moreover, $\tau^{\mathcal{H}}(\mathcal{D})$ can be identified with its image on $M$ by the isometry $T G_{k}(M)^{\mathcal{H}} \rightarrow T M$, and hence it is a $G$-invariant vector field on $M$. Therefore there exists a corresponding $\operatorname{Ad}_{G}(K)$-invariant element in $\mathfrak{m}$, which we again denote by $\tau^{\mathcal{H}}(\mathcal{D})$. We will not distinguish these vector fields because no confusion will be 
caused. We claim that for $M=G / K$ with a metric modified by any $\lambda>0$, this distribution $\mathcal{D}$ defines a harmonic map from $M$ to $G_{k}(M)$.

LEMMA 2. Let $G$ be a compact Lie group with the modified metric $\langle\cdot, \cdot\rangle$ and the LeviCivita connection $\bar{\nabla}$. We then have the following facts about the covariant derivatives of left invariant vector fields in $\mathfrak{m}$.

(1) If $X, Y \in \mathfrak{p}$, or $X, Y \in \mathfrak{n}$, then $\bar{\nabla}_{X} Y=(1 / 2)[X, Y]$.

(2) If $X \in \mathfrak{p}$ and $Y \in \mathfrak{n}$, then $\bar{\nabla}_{X} Y$ and $\bar{\nabla}_{Y} X$ are in $\mathfrak{n}$.

Proof. Since $\langle\cdot, \cdot\rangle$ is $\operatorname{Ad}_{G}(H)$-invariant, for any $V, W \in \mathfrak{g}$ and $X \in \mathfrak{h}$, we have

$$
\langle[V, X], W\rangle=\langle V,[X, W]\rangle .
$$

For any $X, Y \in \mathfrak{p}=\mathfrak{h} \cap \mathfrak{m}$ and $V \in \mathfrak{g}$, by the Koszul formula, we have

$$
\begin{aligned}
2\left\langle\bar{\nabla}_{X} Y, V\right\rangle & =-\langle X,[Y, V]\rangle-\langle Y,[X, V]\rangle+\langle[X, Y], V\rangle \\
& =\langle[X, Y], V\rangle .
\end{aligned}
$$

Similarly, for any $X, Y, V \in \mathfrak{n}$, since $[\mathfrak{n}, \mathfrak{n}] \subset \mathfrak{h}$ and $\mathfrak{n} \perp \mathfrak{h}$, the Koszul formula gives

$$
2\left\langle\bar{\nabla}_{X} Y, V\right\rangle=-\langle X,[Y, V]\rangle-\langle Y,[X, V]\rangle+\langle[X, Y], V\rangle=0 .
$$

Therefore, we have $\bar{\nabla}_{X} Y \in \mathfrak{h}$ for $X, Y \in \mathfrak{n}$ and it is easy to see again by the $\operatorname{Ad}_{G}(H)$ invariance of the metric that $2\left\langle\bar{\nabla}_{X} Y, V\right\rangle=\langle[X, Y], V\rangle$ for $V \in \mathfrak{h}$.

Since the covariant derivative of an invariant vector field with respect to an invariant vector field is again invariant, it is also easy to verify the statement (2) with the Koszul formula and the fact that $[\mathfrak{h}, \mathfrak{h}] \subset \mathfrak{h}$ and $\mathfrak{n} \perp \mathfrak{h}$.

COROLlaRY 1. For $X \in \mathfrak{p}$ or $X \in \mathfrak{n}$, the one-parameter subgroup $\alpha(t)=\exp t X$ is a horizontal geodesic with respect to the Riemannian submersion $\pi: G \rightarrow M$. Furthermore, each element $X \in \mathfrak{p}$ generates a horizontal Killing vector field on $G$.

Proof. For $X \in \mathfrak{p}$ or $X \in \mathfrak{n}$, since $\bar{\nabla}_{X} X=(1 / 2)[X, X]=0$, the one-parameter subgroup $\alpha(t)=\exp t X$ is a geodesic.

A left invariant vector field $X \in \mathfrak{m}$ is certainly a horizontal vector field, and its flow is given by the right multiplication by $\alpha(t)=\exp t X$. Since the modified metric is still $H$-biinvariant, this flow is an isometry as long as $X$ is in $\mathfrak{h}$.

Since the one-parameter subgroup $\alpha(t)=\exp t X, X \in \mathfrak{p}$ or $\mathfrak{n}$, is a horizontal geodesic with respect to the Riemannian submersion $\pi: G \rightarrow M$, its image $\pi \circ \alpha(t)$ is a geodesic in $M$.

We now take a small neighborhood $U$ of 0 in $\mathfrak{m}$, and let $\Sigma=\exp U$ be the $n$-dimensional submanifold near the identity in $G$, where $n=\operatorname{dim}(\mathfrak{m})=\operatorname{dim}(M)$. Let $\left\{\bar{e}_{A}\right\} \subset \mathfrak{m}, 1 \leq A \leq$ $n$, be an orthonormal frame field along $\Sigma$ such that $\left\{\bar{e}_{i}\right\} \subset \mathfrak{p}, 1 \leq i \leq k$ and $\left\{\bar{e}_{\alpha}\right\} \subset \mathfrak{n}$, $k+1 \leq \alpha \leq n$. Note that we are using the same index convention as in Section 3, and this left $G$-invariant frame field is not necessarily tangent to $\Sigma$ but horizontal with respect to the Riemannian submersion. Denote by $\left\{e_{A}\right\}$ the image of $\left\{\bar{e}_{A}\right\}$ by the projection $\pi: G \rightarrow M$. It is then clear that $\left\{e_{A}\right\}$ is an orthonormal frame of $M$ near the point $(e K)$ such 
that $d \pi\left(\bar{\nabla}_{\bar{e}_{A}} \bar{e}_{B}\right)=\nabla_{e_{A}} e_{B}$, where $\nabla$ is the Levi-Civita connection on $M$. All these covariant derivatives of invariant vector fields in $\mathfrak{m}$ have invariant horizontal components in $G$, which correspond to covariant derivatives in $M$ via the projection. We plan to carry out the computation of covariant derivatives in $M$ in terms of horizontal invariant fields in $G$ up to second order.

Proposition 4. For $M$ and $\mathcal{D}$ as above, we have $\tau^{\mathcal{V}}(\mathcal{D})=0$.

Proof. Since $\tau^{\mathcal{V}}(\mathcal{D})$ is G-invariant, it suffices to show this vector vanishes at the point $(e K)$. We take a frame field $\left\{e_{A}\right\}$ as above. Since $\nabla_{e_{A}} e_{A}=0$, by Proposition 3, for any $e_{i}$ we have

$$
\tau^{\mathcal{V}}(\mathcal{D})\left(e_{i}\right)=\sum_{A}\left(\left(\nabla_{e_{A}}\left(\nabla_{e_{A}} e_{i}\right)^{\perp}\right)^{\perp}-\left(\nabla_{e_{A}}\left(\nabla_{e_{A}} e_{i}\right)^{\top}\right)^{\perp}\right)
$$

where $\tau^{\mathcal{V}}(\mathcal{D})$ is identified as an element in $\operatorname{Hom}\left(\mathcal{D}, \mathcal{D}^{\perp}\right) \oplus \operatorname{Hom}\left(\mathcal{D}^{\perp}, \mathcal{D}\right)$.

For the first term in the right hand side of the above equation, we observe that the fibres are totally geodesic and hence for all $e_{j} \in \mathcal{D}$ we have $\left(\nabla_{e_{j}} e_{i}\right)^{\perp}=0$, where $(\cdot)^{\perp}$ denotes as before the projection onto $\mathcal{D}^{\perp}=d \pi(\mathfrak{n})$. Moreover, by Lemma 2 (2), we have $\bar{\nabla}_{\bar{e}_{\alpha}} \bar{e}_{i} \in \mathfrak{n}$, and hence $\left(\nabla_{e_{\alpha}} e_{i}\right)^{\perp}=\nabla_{e_{\alpha}} e_{i}$ is the image of an element in $\mathfrak{n}$. Since $[\mathfrak{n}, \mathfrak{n}] \subset \mathfrak{h}$, we now see that $\left(\nabla_{e_{\alpha}}\left(\nabla_{e_{\alpha}} e_{i}\right)^{\perp}\right)^{\perp}=0$.

For the second term, again by Lemma 2 (2), we see that $\left(\nabla_{e_{\alpha}} e_{i}\right)^{\top}=0$. Since $[\mathfrak{p}, \mathfrak{p}] \subset \mathfrak{h}$, we have $\left(\bar{\nabla}_{\bar{e}_{j}} \bar{e}_{i}\right)^{\top} \in \mathfrak{p}$ and hence for the same reason $\left(\nabla_{e_{j}}\left(\nabla_{e_{j}} e_{i}\right)^{\top}\right)^{\perp}=0$. Therefore $\left.\tau^{\mathcal{V}}(\mathcal{D})\right|_{\mathcal{D}}=0$, and since $\left.\tau^{\mathcal{V}}(\mathcal{D})\right|_{\mathcal{D}^{\perp}}=-\left(\left.\tau^{\mathcal{V}}(\mathcal{D})\right|_{\mathcal{D}}\right)^{*}$, we can now conclude that $\mathcal{D}$ is vertically harmonic.

By Lemma 2, we see that the covariant derivative $\bar{\nabla}_{X} Y$ coincides with the Lie bracket as long as $X$ and $Y$ are both in $\mathfrak{p}$ or $\mathfrak{n}$. For other cases, although it is more complicated, we could still carry out the computation through algebraic operations and it would be possible to write down the curvature of $G$ or $M$. But, for this type of modified metric on $M$, we already have a nice formula for the curvature of $M$.

Lemma 3 ([11], Lemma 3.6). Let $A: \mathfrak{g} \rightarrow \mathfrak{g}$ be a linear transformation such that $\langle X, Y\rangle:=\langle\langle A X, Y\rangle\rangle$. Then the curvature of the invariant metric $\langle\cdot, \cdot\rangle$ on the compact homogeneous space $M=G / K$ is given by

$$
\begin{aligned}
\langle R(X, Y) Z, W\rangle= & \frac{1}{2}\left\{\left\langle\left\langle B_{-}(X, Y),[Z, W]\right\rangle\right\rangle+\left\langle\left\langle[X, Y], B_{-}(Z, W)\right\rangle\right\rangle\right\} \\
& +\frac{1}{4}\left\{\left\langle[X, W],[Y, Z]_{\mathfrak{m}}\right\rangle-\left\langle[X, Z],[Y, Z]_{\mathfrak{m}}\right\rangle-2\left\langle[X, Y],[Z, W]_{\mathfrak{m}}\right\rangle\right\} \\
& +\left\{\left\langle\left\langle B_{+}(X, W), A^{-1} B_{+}(Y, Z)\right\rangle\right\rangle-\left\langle\left\langle B_{+}(X, Z), A^{-1} B_{+}(Y, W)\right\rangle\right\rangle\right\},
\end{aligned}
$$

where $B_{+}(X, Y)=(1 / 2)([X, A Y]+[Y, A X]), B_{-}(X, Y)=(1 / 2)([A X, Y]+[X, A Y])$, and $[\cdot, \cdot]_{\mathfrak{m}}$ denotes the $\mathfrak{m}$-component. 
Note that in the above formula for the curvature of $G / K$ the second line with the $\mathfrak{m}$ components accounts for the O'Neill's term of the Riemannian submersion $G \rightarrow G / K$, and all the computations are done in $\mathfrak{g}$ with invariant vector fields in $\mathfrak{m}$.

COROLlary 2. For all $W \in \mathcal{D}^{\perp}$, we have $\left\langle\tau^{\mathcal{H}}(\mathcal{D}), W\right\rangle=0$. In particular, $\tau^{\mathcal{H}}(\mathcal{D}) \in$ $\mathfrak{p .}$

Proof. Let $\left\{e_{A}\right\}$ be the same local orthonormal frame field of $M$ as in Proposition 4. Since the fibres are totally geodesic, for all $e_{i}, e_{j} \in \mathcal{D}$ we have $\left(\nabla_{e_{j}} e_{i}\right)^{\perp}=0$. Thus by Proposition 2,

$$
\begin{aligned}
\tau^{\mathcal{H}}(\mathcal{D}) & =\sum_{i, A} R\left(\left(\nabla_{e_{A}} e_{i}\right)^{\perp}, e_{i}\right) e_{A} \\
& =\sum_{i, \beta} R\left(\left(\nabla_{e_{\beta}} e_{i}\right)^{\perp}, e_{i}\right) e_{\beta} \\
& =\sum_{\alpha, \beta} R\left(\left(\nabla_{e_{\beta}} e_{\alpha}\right)^{\top}, e_{\alpha}\right) e_{\beta}=\tau^{\mathcal{H}}\left(\mathcal{D}^{\perp}\right) .
\end{aligned}
$$

The Riemannian metric on $M=G / K$ is determined by $\langle\cdot, \cdot\rangle$ with $\left.\langle\cdot, \cdot\rangle\right|_{\mathfrak{h}}=\lambda\langle\langle\cdot, \cdot\rangle\rangle$, that is, $\left.A\right|_{\mathfrak{p}}=\lambda \cdot \operatorname{Id}$ and $\left.A\right|_{\mathfrak{n}}=\operatorname{Id}$. For any $X \in \mathfrak{p}, Y, Z, W \in \mathfrak{n}$, we obtain

$$
B_{-}(X, Y)=\frac{1}{2}([A X, Y]+[X, A Y])=\frac{1}{2}(\lambda+1)[X, Y],
$$

and similarly we have $B_{-}(Z, W)=[Z, W], B_{+}(X, W)=(1 / 2)(1-\lambda)[X, W], B_{+}(Y, W)$ $=B_{+}(Y, Z)=0$. Since, $[\mathfrak{n}, \mathfrak{n}] \subset \mathfrak{h}=\mathfrak{k} \oplus \mathfrak{p},[\mathfrak{h}, \mathfrak{n}] \subset \mathfrak{n}$ and $[\mathfrak{p}, \mathfrak{n}] \subset \mathfrak{n}$, by Lemma 3, we have

$$
\begin{aligned}
\langle R(X, Y) Z, W\rangle= & \frac{1}{4}(3+\lambda)\langle\langle[X, Y],[Z, W]\rangle\rangle \\
& +\frac{1}{4}\left\{\left\langle[X, W],[Y, Z]_{\mathfrak{m}}\right\rangle-\left\langle[X, Z],[Y, W]_{\mathfrak{m}}\right\rangle\right. \\
& \left.-2\left\langle[X, Y],[Z, W]_{\mathfrak{m}}\right\rangle\right\}=0 .
\end{aligned}
$$

Put $X=\left(\nabla_{e_{\beta}} e_{\alpha}\right)^{\top}, Y=e_{\alpha}, Z=e_{\beta}$. Then we have $\tau^{\mathcal{H}}(\mathcal{D}) \in \mathcal{D}$. Since $\tau^{\mathcal{H}}$ is a $G$-invariant vector field tangent to $\mathcal{D}$, we conclude that $\tau^{\mathcal{H}}(\mathcal{D})$ is an $\operatorname{Ad}_{G}(K)$-invariant element in $\mathfrak{p}$ by the canonical identification of $T_{p} M$ with $\mathfrak{m}$.

By the above corollary, we see that the $\mathfrak{n}$-component of $\tau^{\mathcal{H}}(\mathcal{D}) \in \mathfrak{m}$ vanishes. However, the $\mathfrak{p}$-component can not be controlled in this way and we need a direct approach.

Proposition 5. For $M$ and $\mathcal{D}$ as above, we have $\tau^{\mathcal{H}}(\mathcal{D})=0$.

Proof. It suffices to show that $\tau^{\mathcal{H}}(\mathcal{D})=0$ at $(e K)$. Let $f: M \rightarrow G_{k}(M)$ be a section given by the distribution $\mathcal{D}$, and let $\Sigma:(-\varepsilon, \varepsilon) \times M \rightarrow G_{k}(M)$ be a horizontal variation such that $\Sigma(0, p)=f(p),(\partial \Sigma / \partial t)(0, p)=\tau^{\mathcal{H}}(\mathcal{D})$, and $f_{t}(p):=\Sigma(t, p)$. Along $f$ the horizontal vector field $\tau^{\mathcal{H}}(f)$ is a horizontal lift of its image on $M$, which we do 
not distinguish. Denote by $\varphi_{t}$ the flow of $\tau^{\mathcal{H}}(f)$ on $M$, i.e., $\varphi_{t}=\pi \circ f_{t}$. Since $\tau^{\mathcal{H}}(f)$ is lifted to an $\operatorname{Ad}_{G}(K)$-invariant vector field $\tau^{\mathcal{H}}(f) \in \mathfrak{p}$ on $G$ with respect to the Riemannian submersion $G \rightarrow G / K$, by Corollary 1 , it is a Killing field on $G$. Furthermore, the image of this horizontal vector field by the projection $\pi: G \rightarrow M$ gives rise to a Killing vector field $\tau^{\mathcal{H}}(f)$ on $M$.

Since $t \mapsto f_{t}(p)$ is a horizontal lift of the curve $t \mapsto \varphi_{t}(p)$, it represents the parallel transport of $f(p)$ along the curve. Furthermore, by Corollary 1 , for $p=(e K) \in M$ the flow $\varphi_{t}(p)=\pi \exp \left(t \tau^{\mathcal{H}}(f)\right)$ is a geodesic and hence is contained in a totally geodesic fibre. Therefore the tangent space of fibre is invariant under the parallel transport along the flow. Thus we have $f_{t}=f \circ \varphi_{t}$. Since $\varphi_{t}$ is an isometry for each $t$, we see that $\left\|d f_{t}\right\|^{2}$ is a constant. Therefore

and hence $\tau^{\mathcal{H}}=0$.

$$
\int_{M}\left\langle\tau^{\mathcal{H}}, \tau^{\mathcal{H}}\right\rangle d v=-\left.\frac{d}{d t}\right|_{t=0} E\left(f_{t}\right)=0
$$

Combining Propositions 4 and 5, we now have the following.

THEOREM 2. Let $M$ be a manifold constructed as above. Then the G-invariant distribution $\mathcal{D}$ is a harmonic map into the Grassmannian bundle $G_{k}(M)$.

As we mentioned, all of the generalized Hopf-fibrations are constructed in this way, and hence distributions tangent to the Hopf fibre are harmonic maps into the Grassmannian bundle.

\section{REFERENCES}

[1] A. L. Besse, Einstein manifolds, Springer-Verlag, Berlin, 1987.

[ 2 ] J. Eells AND L. Lemaire, A report on harmonic maps, Bull. London Math. Soc. 10 (1978), 1-68.

[ 3 ] J. Eells AND L. Lemaire, Another report on harmonic maps, Bull. London Math. Soc. 20 (1988), 385-524.

[ 4 ] R. Escobles, Riemannian submersions with totally geodesic fibres, J. Differential. Geom. 10 (1975), 253276.

[ 5 ] H. Gluck AND W. Gu, Volume-preserving great circle flows on the 3-sphere, Geom. Dedicata 88 (2001), $259-282$.

[ 6 ] D.-S. HAN AND J.-W. YIM, Unit vector fields on sphere, which are harmonic maps, Math. Z. 227 (1998), 83-92.

[ 7 ] T. IshiHARA, Harmonic section of tangent bundles, J. Math. Tokushima Univ. 13 (1979), 23-27.

[ 8 ] G. Jensen AND M. Rigoli, Harmonic Gauss maps, Pacific J. Math. 136 (1989), 261-282.

[ 9 ] J. J. KonderaK, On harmonic vector field, Publ. Mat. 36 (1992), 217-228.

[10] B. O’NEILL, The fundamental equations of a submersion, Michigan Math. J. 13 (1966), 459-469.

[11] T. PÜtTmann, Optimal pinching constant of odd dimensional homogeneous spaces, Invent. Math. 138 (1999), 631-684.

[12] H. URaKawa, Calculus of Variations and Harmonic Maps, Transl. Math. Monogr, 132, American Mathematical Society, Province, RI, 1993.

[13] C. M. Wood, The Gauss section of a Riemannian immersion, J. London Math. Soc. (2) 33 (1986), 157-168.

[14] Z. ZHANG, Best distributions on Riemannian manifolds and elastic deformations with constant principal strains, Ph. D. thesis, University of Pennsylvania, 1993. 
188

DEPARTMENT OF MATHEMATICS AIR FORCE ACADEMY

SSANGSU, NAMiL, CheONGWon

ChungBuK, 363-849

SOUTH KOREA

E-mail address: bychoy@ afa.ac.kr

\section{B.-Y. CHOI AND J.-W. YIM}

DEPARTMENT OF MATHEMATICS

Korea Advanced Institute of Science and Technology GU-SUNG DONG, YU-SUNG GU

TAEJON, 305-701

KOREA

E-mail address: jwyim@math.kaist.ac.kr 